\title{
Mécanique des terrains perméables
}

\section{The mechanics of permeable soils}

\author{
IAR J. FELRANDON
}

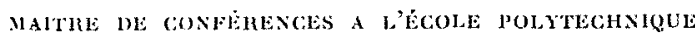

\begin{abstract}
Le texte ci-dessous résume la matiere d'me série de conférences faites aux Elènes Inginieurs de l'Institut Polytechnique de Grenoble alu cours de ta session scolaire 1953-195\%. Leur objet est lexamen de cerlaines propriétés des terrains permécables particalierement uliles pour les applications.

la premiere partie expose les lois de l'éconlement laminaire en généralisant, par l'introduction du tenseur de perméabilite, la loi de Darcy anx milieux isotropes.

La seconde partie traite de l'equilibre limile des sols cohérents sans frotlement interne et des sols pulvérulents par la inéthode analytique de Cauchy, par laquelle les lignes de glissement apparaissent comme courbes caractéristiques d'un système d'équation aux dérivées partielles.

La troisième partie envisage les phénomènes de propagation de discontinuités dans un sol perméable, compte tenu de l'existence de denx phases solide ef liquide en présence.

Enfin whe note de M. F. Serre sur l'solution en fonction du temps dil tassement des couches argileuses aborde le problème du tassement des fondations d'une munière toat à fail générale.
\end{abstract}

\begin{abstract}
The following text is a resume of a series of lectures delinesed to engineering students at the Grenoble Polylechnic Institute during the 1953-1951 session. The object of the lectures mas to examine those properties of permeable soils which are of special importance in practical problems.

The first part treats the laws of laminar flow, generalising Darcy's law for isolsopic soils by inlroducing the permeability tensor.

The second part deals with the limiting equilibrium of cohesive soils without internal friction, and of poudery soils. The analytical method of Cauchy is used and gives the failure lines as characteristic curves of a system of partial differential equations.

The third part considers the phenoment of propagation of aiscontinuities in a permeable soil taking into account the existence of both solid and liquid slages.

linally a note by M. F. SEнus on the time clement in the settlement of clay strala introda. ces the problem of foundalion selllement in a very genteral manner.
\end{abstract}

\section{PREMIERE PARTIE}

\section{ÉCOULEMENT DE L'EAU DANS LES TERRAINS PERMEABLES}

Li Mécanique des sols comporte l'étude des phénomènes de filtration dont les massifs naturels sont généralement le siège et pour laquelle est essentielle la connaissance des lois régissant l'cooulement de l'eau au travers des milieux perméables.

La premiere partie de cet ouvrage consiste (n un exposé analytique de ces lois sous forme suffisamment générale pour engigher la multiplicité des cas usuels et suffisamment précise pour en permeltre la résolution. Y est constamment envisagé un massil naturel poreux formé de grains solides ménageant entre eux des intervalles tris petits dont l'ensemble constitue un réseau de canaux ou tubes de liltration de tris faibles sections imprégnés d'eau. Toule différence de charge entre deux régions du liquide provoque un lent mouvement de filtration. Dans chacun des tubes, le mouvement de l'eau se comporte comme celui d'un liquide visqueux à l'intérieur d'un tube fin avec vitesse nulle au contact des parois.

Les propriétés de ce cas élémentaire et fondamental sont donc exposées en premier lieu pour che dendues sucessivenent a ceux de la fill ration unidimensionnelle puis de la plus générale, c'est-à dire sans direction privilégiée d'écoulement. Dinns ces diverses eventualités sont effecluées la détermination du champ des vitesses de l'eau et celle des actions hydrodynamiques exercćes sur les grains du massif filtrant.

Ces conférences relatives a la Mécanique des sols perméables comportent trois parties: 
1. -...- La filtration d'un liquide visqueux à l’inlerieur d'un sol poreux à slruelure invariable. esl d'abord cxamince. Sont mises en évidence ha loi de filtration el déterminces les actions du liquide filtrant sur la phase solide.

II. - Le chany de ces actions s'ajoute à celui fe la pesanteur el modifie les conditions dépuilibre des massifs ou intervicnnent tels coonlements de l'espece. Sont examinces diverses circonslances de rupture par glissement de ceux-ci.

III. - Si le sol est susceptible de diformation propre - argiles - les actions en cause modifiant l'état des forces intérieures de la pliase solide en provoquent l'évolution. Sont étudiés deux éventualités extrêmes de celle-ci : la propagation par ondes d'excitations inilialement localisées sur la strdace limitant le massif el l'évolution lente ou consolidation.

Ie lexte ci-riessous comporte les calculs qu'il n'a pas élé possible de développer à lamphithiblite.

\section{I}

\section{ÉCOULEMENT GRADUELLEMENT VARIÉ DANS UN TUBE FIN}

1.

L'étude de l'écoulement graduellement varic dans un tube fin, déduit des équations générales de l'hydrodynamique des fluides visqueux incompressibles, conslitue lexposé préliminaire indispensable à celle de la filtration au lravers des matssifs. Les résultats qui y sonl oblenus s'étendent assez directement aux siluations nalurolles plus complexes et les approximations consentics qui y sont mises en ividence précisent le sens et limitent la portée de leur application.

\section{EQUATIONS DE MOUVEMENT.}

Est donc envisagé un lube eylindrique à generatrices paralleles à ox, inclinécs sur l'horizon da l'angle $i$, dont la section droite du plan yoz (oz horizonlale) a pour are $\omega$ el pour périmetre $\%$ (soction el périmetre mouillés) (fig. 1 ).

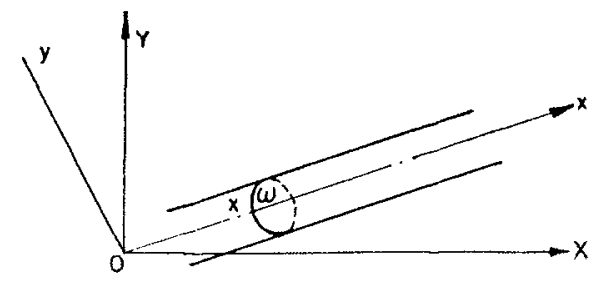

Fiti. 1 .
Le liguide, de poids specilique $\bar{x}: \ldots y$, de coellicient cinématique de viscosite $v: \ldots, 0 / 0$, qui to remplit, sy écoule sous laction de la pesamben d'accéléation $g$, arece en lout point $(x, y, z)$ el a l'instant $t$, la vilesse $V(x, y, z, t)$ de coordonnies a, $b$, w). Des simplificalions chassiques qui conduisent a supposer mulles dans lout le liquide les coordonnces $l$ el $b$ de sa vilesse ansi que les dérivées parlielles $\partial u / \partial x^{*}$ el $\partial " u / \partial x^{2}$, permettent. en designant par $p(x, y, z, t)$, la pression reversible all point $(x, y, z)$ el à l'instant / de meller les iquations de lhydrody namique propres atux écoulements de l'espoec subs la forme!" :

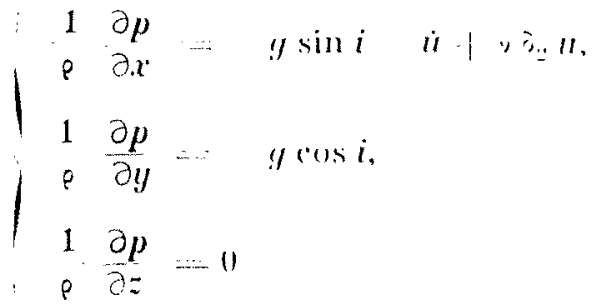

Sy adjoinl ta condilion anx limiles:

$u:=0$, sur lat surlate laterale du tube.

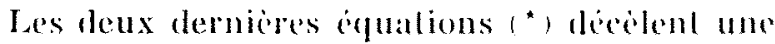
distribution hydroslutique des pressions dans coule section drote du lube.

La fremiere est susceptible de suggestives Iransformations.

Lintroduction dans cellerei de la pente metrice :

$$
1-\left|\sin i \cdot \begin{array}{cc}
\partial & \mu \\
\partial x & \frac{1}{\pi} !
\end{array}\right|
$$

domne: :

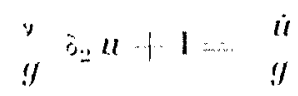

suil. Har integration a la section momilled dairs (1) :

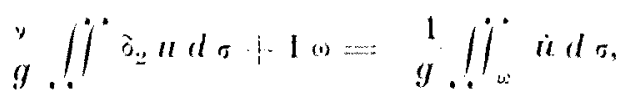

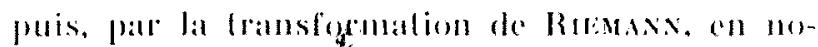

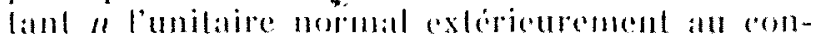
low $\%$ :

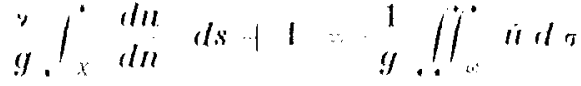

$$
\begin{aligned}
& \text { 'i On a posé : }
\end{aligned}
$$

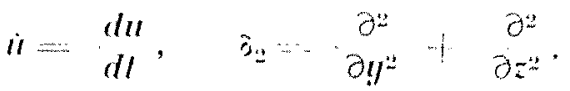


enfin, par élimination de I entre (t) et cette dernière :

$$
\vee \delta_{2} u-\frac{v}{\omega} \int_{x} \frac{d u}{d n} d s=\dot{u}-\frac{1}{\omega} \iint_{\omega}^{+} \dot{u} d \sigma(6)
$$

A cette équation s'ajoute la condition aux limites (2), et le groupe ainsi formé définissant complètement le mouvement axial envisagé par la prise en considération de la vitesse moyenne:

$$
\mathrm{L}=\frac{1}{\omega} \iint_{\omega}^{\infty} u d \sigma
$$

peul sécrire :

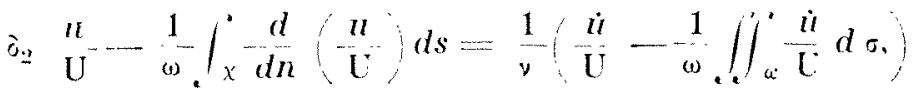

$$
\begin{aligned}
& \frac{1}{\omega} \iint_{\omega} \frac{u}{\mathrm{U}} \cdot d \sigma=1 \\
& \frac{u t}{\mathrm{U}}=0 \text { sur la surface latérale du tube. }
\end{aligned}
$$

Pour tout mouvement graduellement varic, c'est-à-dire demeurant en bref assez proche d'un mouvement uniforme, le système intégro-différentiel $(8)$, apparemment fort complexe, est jusliciable d'une fructueuse méthode d'approximalion qui va être exposée.

\section{DEFFNITION D'UN MOUVEMLNT ENHOHME DE RÉFÉRENCE.}

Si l'écoulement est unifornte, $\dot{u}=0$. Posant $u / U=\zeta$, le systime (8) se simplific et devient :

$$
\left\{\begin{array}{l}
\delta_{2} \xi-\frac{1}{\omega} \int_{x} \frac{d \xi}{d n} d s=0 \\
\frac{1}{\omega} \iint_{\omega} \zeta d \sigma=1 \\
\zeta=0 \text { sur la surface latérale du tube. }
\end{array}\right.
$$

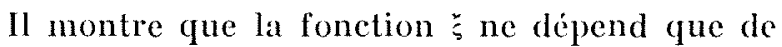
la section $\omega$.

Pour un tube circulaire de rayon $r$, par exemple, une intégration classique donne :

$$
\xi=2\left(1-\frac{y^{2}+z^{2}}{l^{2}}\right)
$$

Cetle constatation suggere, afin de poursuive l'élude du mouvement graduellement varić, de la rapporter à un mouvement uniforme de référence de mème débit, c'est-à-dire de même vitesse $U$, el de fixer son état par l'écart de vilesse, ou par loule quantité proportionnelle à celle-ci, qu'il présente avee ce dernier. D’une façon précise, à cet effet, est choisi le nombre :

$$
\varepsilon=\frac{u}{\mathrm{U}}-\xi
$$

u est la vitesse réelle du mouvement graducllement varié, $U$ sa vitesse moyenne dans la section $\omega, 5$ la valeur du quotient $u / U$ de la vilesse du mouvement uniforme de vitesse movenne. $U$. Par hypothese, ₹ demeure petil devant $u / \mathrm{U}$.

\section{LOI DU MOUTEMENT GRADUELLEMENT VARHÉ.}

Comme conséquence directe des définitions posées el des résultats obtenus ci-dessus, l'écarl $\varepsilon$ satisfait au système d'équalions :

$$
\left\{\begin{array}{l}
\delta_{.2} \varepsilon-\frac{1}{\omega} \int_{\chi} \frac{d \varepsilon}{d n} d s=\frac{1}{\nu \mathrm{U}}\left(\dot{u}--\frac{1}{\omega} \iint_{\omega} \dot{u} d \sigma\right) \\
\iint_{\omega} \varepsilon d \sigma=0 \\
\varepsilon=0, \text { sur la surface latérale du tube }
\end{array}\right.
$$

analogue à (8), mais le degré d'infinilude de $\varepsilon$ permet d'en simplifier le développement,

Multipliant les premières équations (9) et (11) respectivement par - $-\varepsilon d \sigma$ et $\varepsilon d \sigma$, ajoutant, puis intégrant dans $\omega$, après aroir désigné par $\lambda$ la quantité.

$$
\lambda=\cdots \int_{x}^{\cdot} \frac{d \varepsilon}{d n} d s
$$

dependant comme \% de lat seule section (1) , il vient :

$\int_{\chi}^{\cdot} \frac{d u}{d n} d s+\lambda \mathrm{U}+\frac{1}{\nu} \iint_{\omega}^{\infty}(\varepsilon-\cdots 1) \dot{\|} d \sigma=0 .($

L'élimination de l'intégrale curviligne entre les equations (5) el (13) donne:

$$
\mathrm{I}=\frac{\lambda y}{g \omega} \mathrm{U}+\frac{1}{g \omega} \iint_{\omega}^{\nu} \varepsilon \dot{u} d \sigma
$$

Ici intervient lapproximation annoncée tenanl a la pelitesse de $\varepsilon$. Par (10), en ellel, le second lerme de (14) égal à :

$$
\frac{1}{g \omega} \cdot \int_{\omega}^{*}\left(\begin{array}{l}
\| \\
U^{-\cdots}
\end{array}\right) \dot{l} d \sigma
$$

est équivalent à :

$$
\frac{1}{g \omega} \iint_{\omega}^{*} \frac{u \dot{u}}{\mathrm{U}} d \sigma
$$


et l'évaluation de cette sommation qui s'effectue en posant :

$$
\alpha={ }_{\omega}^{1} \int_{\omega}^{*}\left(\frac{u}{U}\right)^{:} d \sigma
$$

donne :

$$
\frac{\partial}{\partial x}\left(\alpha \frac{\mathrm{U}^{2}}{2 y}\right)
$$

de lelle sorle que :

$$
\mathrm{I}=\frac{\lambda \nu}{g \omega} \cdot \mathrm{U}+\frac{\partial}{\partial x}\left(\alpha \frac{\mathrm{U}^{2}}{2 g}\right)
$$

Enfin, par la définition (3) de I el en faisant ctat de l'égalité de $\sin i$ à $\partial Y / \partial x$ (OY verticale ascendante), il vient l'equation du mouvement graduellement varié :

$$
\frac{\lambda \nu}{g \omega} \mathrm{U}+\frac{\partial}{\partial x}\left(x \frac{\mathrm{U}^{2}}{2 g}-\frac{p}{\omega}+\mathrm{Y}\right)=0
$$

qui affirme la proportionnalité de la vitesse moyenne d'écoulement $\mathrm{U}$ au gradient de charge hydraulique :

$$
\Phi=\frac{\alpha U^{2}}{2 g}+\frac{p}{\sigma}+\mathrm{Y}
$$

Dorénavant, seuls seront pris en considération les écoulements à très faibles vitesses, pour lesquels $\alpha \mathrm{U}^{2} / 2 g$ est négligeable devant $(p / \pi)+$ Y. lans ces conditions, la charge réduite à ses lernes de pression et de position est équivalente à :

$$
\Phi=\frac{p}{\omega}+\mathrm{Y}
$$

qui sera retenue pour representer la vilesse noyenne d'écoulement par :

$$
\mathrm{U}=-\frac{g \omega}{\lambda y} \frac{\partial \Phi}{\partial x} .
$$

Les conséquences de (19) qui seront décrites ont donné lieu à des vérifications expérimentales généralement satisfaisantes qui justifient les approximations ci-dessus, effectuces sous la double hypothese diun mouvement quasi aniforme et tres lent.

\section{j. Acrow sun La TCBE.}

Le tube considéré en (2) est limité par sa surface latérale cylindrique (L) et par deux sections planes non nécessairement droiles $(\sigma)$ et $\left(\sigma^{\prime}\right)$. Soil $\pi(\alpha, \beta, \gamma)$ la normale extérieure en tout point regulier de ce contour (fig. 2).

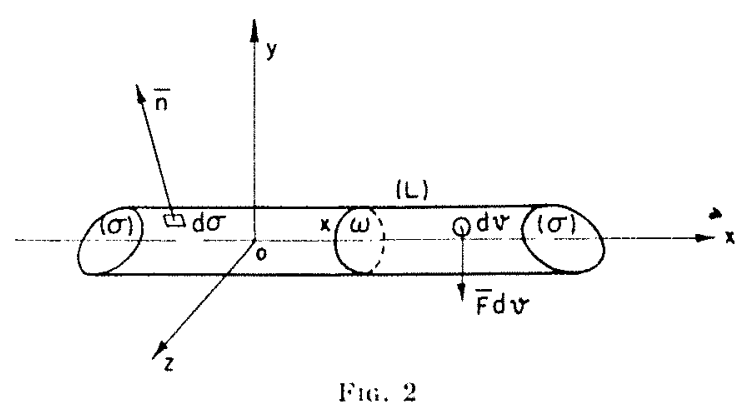

Une particule de volume d $\vartheta$ du liquide fill rant a la vilesse $\vec{V}$ est sollicite par la lorec de pesanleur Fd $v$, dont le polentiel est designe par $Y(F=-$ grad $r)$. Les eflorts a la surface du liquide contenu dans le lube, opposés à ceux exerces par celui-ci, tant sur la paroi (L) du lube que sur les sections liquides $(\sigma)$ ef $\left(\sigma^{\prime}\right)$, sont designés par T $d \sigma$ pour tout dément d’aire $d \sigma$, de telle sorle que l'aclion globale a appliqué ì (L) est :

$$
a=-\iint_{\mathrm{L}}^{\mathrm{T}} \mathrm{T} d \sigma
$$

Le Theoreme des quantites de mombement en permet la détermination. Pour le liquide contenu dans le tuhe, il s'ecrit en effet:

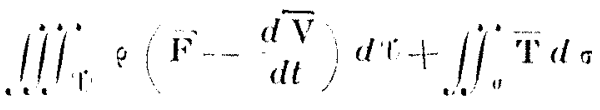

$$
\begin{aligned}
& +1 \ddot{j}_{\sigma} \mathrm{T} d \sigma+1 \ddot{\mathrm{j}} \mathrm{T} d \sigma=0
\end{aligned}
$$

d'où :

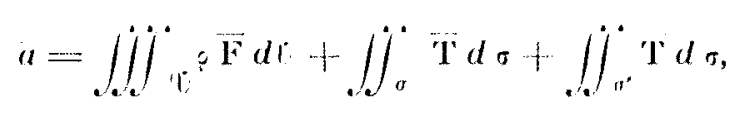

en négligeant les forces d'inertie devant les forces données, comme, et pour la même raison, l'energie cinétique du filet liquide a ché négligée en [4] dans l'expression de son énergic totale.

Or, le vecteur $\mathrm{T}$, produit par $\pi$ du lenseur-tension $\overrightarrow{\mathrm{T}}$ de coordonnces (*):

$$
\begin{aligned}
& \mathrm{N}_{1}=\mathrm{N}_{2} \cdots \mathrm{N}_{3}=\cdots l \\
& \mathrm{~T}_{1}=0, \quad \mathrm{~T}_{2}=\frac{\partial u}{\partial z}, \quad \mathrm{~T}_{: 3}=\frac{\partial u}{\partial !},
\end{aligned}
$$

*) Nolation de Lanó.

On a posé : $\mu=v_{0}$.

Compta tenu de ce que $b=w=0,2 !$. 
a pour composantes:

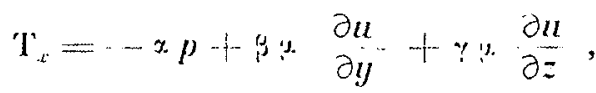

$$
\begin{aligned}
& r_{l y}=x: \begin{array}{l}
\partial u \\
\partial y-\beta p
\end{array} \\
& \mathrm{H}_{z}=x y . \quad \partial u--\eta \mu
\end{aligned}
$$

el il vient pour celles de $\bar{a}$ :

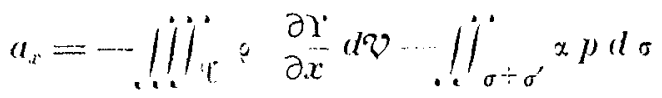

$$
\begin{aligned}
& +\because \int \prod_{\sigma+\sigma^{\prime}} \frac{\partial u}{\partial y}+\gamma \frac{\partial u}{\partial z} d \sigma,
\end{aligned}
$$

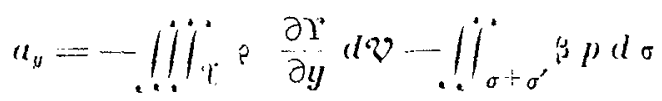

$$
\begin{aligned}
& +\iint_{\sigma \rightarrow \sigma^{\prime}}^{\alpha} \frac{\partial u}{\partial ! j} d \sigma, \\
& a_{z}=-\|\ddot{\varphi}\|_{\varphi} \frac{\partial \gamma^{2}}{\partial z} d \vartheta-\ldots-\| \dot{\varphi}_{\sigma+\sigma^{\prime}} \gamma \nu d \sigma \\
& +\because \int_{\sigma+\sigma^{\prime}} \alpha \frac{\partial u}{d z} d \sigma
\end{aligned}
$$

Mais il est aisé de sassurer, par transformation en intégrales curvilignes, que les derniers termes de chacune des expressions précédentes sont nuls comme est nul $u$ sur les périmètres de $\sigma$ et $\sigma^{\prime}$, si bien que l'action cherchée est équipollente au vecleur (**) :

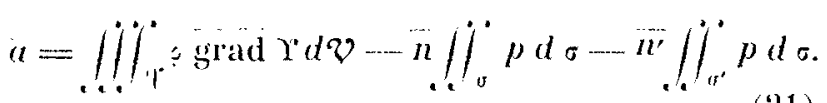

Le fait que celle grandeur soit independante de la viscosité du liquide est lout à fail remarquable. Il est à l'origine d'utiles simplifications dans l'evaluation des efforts exercés sur un massil perméable par le liquide qui s'y écoule.

\section{II}

\section{FILTRATION UNIDIMENSIONNELLE}

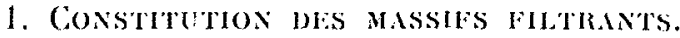

Les sols perméables (sables, argiles, vases) sont constitues essentiellement par fa juxtaposilion de grains solides, enrobés d'eau qui y adhere plus ou moins, et de ce fait ne participe pas atu mourement géneral de filtration, ménageant en-

\footnotetext{
(*) $n$ et $n^{\prime}$, unitaires normaux exterieurement a
} it $\left.\sigma^{\prime}\right)$. tre eux des intervalles actifs gorgés d'eau qui s'y écoule. Sera envisagée une fragmentation de leils ensembles en particulier macroscopiques, de volume considéré comme infinitésimal pour les besoins de l'analyse, mais comportant un lres srand nombre de grains et par suite un très grand nombre d'intervalles aclifs. La surface limilant une telle particule est, par définition de celle-ci, lout entiòre tracée dans les seuls intervalles actifs, dohappant ainsi aux grains of aux pellicules deau qui y demcurent attaches.

les intervalles actif's s'assemblent en tubes de fillration et il est ici supposé que ceux-ci sont sensiblement parallèles à l'axe.

\section{LOI DE LA FILTRATION. \\ COLIFICIENT DE PERMÉABLITÉ.}

Pour chacun de ces tubes, il est loisible de conceroir des caractéristiques géométriques moyennes et notamment une section droite d'aire $\omega$, de périmelre $\%$, à travers laquelle fillre le débit (1.1.19):

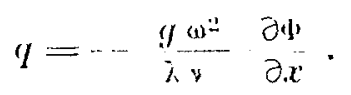

Soit alors une section d'aire 4, normale an tube sur laquelle, par conséquent, le gradient hydraulique $\partial \Phi / \partial x$ demeure constant et dont la scrtion monillée, c'est-à-dire l'aire totale des sections droites des intervalles actifs coupés par (Q) est $r_{1} \Omega$. Le dibil $Q$ à lravers (Q) est :

$$
\mathrm{Q}=-\frac{g}{v} \sum_{0} \omega^{2} \cdot \frac{\partial w}{\partial x}
$$

La vitesse moyenne nelle, rappotice aux seuls tubes de fillration, est :

$$
\mathrm{U}=\frac{\mathrm{Q}}{\mathrm{n} Q}
$$

La vilesse moyenne brule, relalive a la seclion liltrante d'aire $Q$, est :

$$
V^{\prime}=\frac{Q}{\Omega}
$$

Ces deux srandeurs ne doivent faire l'objel d'aucune confusion. Dexperiences systematiques sur des filles de sable, Donky a pu dedure la proportionnalité de la vitesse moyenne brule de fillration $\mathrm{U}$ al gradient hydratuligue $\partial \mathrm{g} / \partial \mathrm{x}$. Celle Ioi :

$$
V=-K \begin{aligned}
& \partial d \\
& \partial x
\end{aligned}
$$

est bien compatible avec les equations de l'hydrodynamique comme il résulte des développe- 
ments précédents qui mettent en outre en lumière la dépendance du coefficient de perméabilité $\mathrm{K}$ ayant les dimensions ( $\mathrm{L}^{\mathrm{r}} \mathrm{T}^{-1}$ ) d'une vitesse des caractéristiques physiques du filtre el du liquide filtrant. En effet :

$K=\frac{g}{\Omega_{\nu}} \sum_{0} \frac{\omega^{2}}{\lambda}=\frac{g \eta_{\nu}}{\Omega_{\nu}} \times$ valeur moyenne de $\frac{\omega}{\lambda}$ dans $\Omega$.

Ii est ainsi fonction des dimensions transversales et de la forme des tubes $(\omega / \lambda)$, de la distribution de ces tubes $\left(r_{1}\right)$, de la viscosile de l'eau (v).

Pour simplifier l'écriture de certains développements ultérieurs, nous posons:

$$
l=\frac{g}{y} \text { valeur movenne de: } \frac{\omega}{\lambda}
$$

si bien que :

$$
K=r_{i}
$$

Celte analyse, qui explicite les divers facteurs dont dépend la perméabilité d'un filtre, n'est guère susceptible, ètant donné l'irrégularité de forme des tubes et la diversité de leur répartition dans un sol donné, d'extension quantitative, el c'est à l'expérimentation, sur échantillon prélevés ou mieux in situ, que sont demandées en fait et pour chaque cas particulier les valeurs numériques du coefficient de perméabilité dont voici, ci-dessous, pour diverses natures de lerrains, quelques ordres de grandeur.

\section{VALEURS MOYENNES APPROXIMATIVES DU COLFFICIENT DE PERMÉABHLITÉ POUR DIVERS TERRAINS}

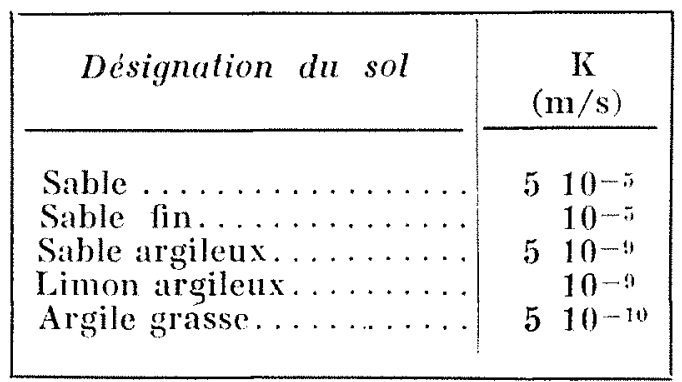

\section{ACTION DE COURANT.}

Soil une fraction finie d'un massif, occupant, srains et liquide compris, le volume $\mathfrak{v}$ limite par la surface $\mathcal{S}$ et comportant un tris grand nombre de grains dont les intervalles actifs de volume total $\mathfrak{V}^{\prime}$ sont gorgés d'eau en mouvement ou susceptibles de se mouvoir sous une difre- rence de charge convenable. Le module des vides $\left(^{*}\right)$ de ce milieu étant $m$ par définition :

$$
v^{\prime}=m v
$$

La résultante $\vec{A}$ de l'action de l'eau sur l'ensemble des grains de $\mathfrak{Y}$ se déduit de (1.1.21) par une double integration etendue :

- Au volume $\vartheta^{\prime}$ occupé effectivement par l'au libre ;

- A la surface $\mathcal{S}$, car sur celle-ci l'aire des grains ou de la pellicule d'eau fixée à chacun d'eux est nulle ou quasi nulle.

Il vient :

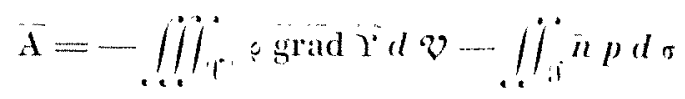

Or, par la transformation de GRlix :

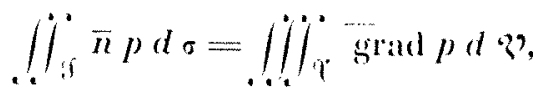

et pour le volume infinitésimal d $\%$ eomportant le volume d'eat $d \vartheta^{\prime}=m d ?$, l'atclion élémenlaire sur les grains est :

$$
\left.d \mathrm{~d}=-(0 m \text { grad })^{2}+\text { grad } p\right) d \eta
$$

Dans le champ gravitique (OY verticale ascendante), $\mathrm{x}=g \mathrm{Y}$, el comme la charge de est équivalente à $(p / \bar{\omega})+\mathrm{Y}$, introduisanl dans $(8)$ celte derniere quantité, celle-ci prend la forme:

$d \mathrm{~A}=\mid-\bar{g}$ grad $\vec{b}+\bar{\omega}(1-m)$ grad $\mathrm{Y} \mid d \vartheta(9)$

Le second terme de celle somme représente laction archimedienne sur le volume $(1-3-m)$ a? des grains solides contenus dans $d \mathcal{V}$, a laquelle se reduit l'action de l'eau en l'absence d'écoulement, puisque ha charge d est alors constante dans lout le massif.

liaction de courant correspond ainsi au premier terme de (9), soil - $-\pi$ grad di $/ v$. Son caractère de force de volume mérile d'ètre noté.

\section{III}

\section{FILTRATION TRIDIMENSIONNELLE}

1.

Comme all chapilte précedent, il est suppose que les inlervalles du massif s'assemblent natu-

") Le module des pides ne doil pas ctre confondu arec l'indice des vides a frequemment utiljé pat les prati. ciens, defini comme rappert du volume des vides at volume des pleins et lie au précedent par $+(m / 1-m)$. 
rellement en tubes de filtration dans lescuuels leau s'écoule effectivement. Mais ici, ces derniers sont a priori de directions quelconques dans l'espace. Cette organisation contient un schéma de calcul propice à de fructueuses investigations analytiques qui vont ètre exposées.

\section{LOI DE LA FILTRATION. TENSEUR DE PERMEABI-} LITÉ.

Soient $\mathrm{M}(x, y, z)$ un point du massif filtrant rapporté a trois axes de coordonnées rectangulaires $O x, O y, O z$ (Oy verticale ascendante) el en ce point, tracé dans le massif, l'élément de surface $\sigma$ de normale $n(\alpha, \beta, \gamma)$ et l'angle solide infiniment délié $d \Sigma$, de sommet $M$, d'axe $\pi$ (fig. 3). Soit $\eta_{i}(\bar{n}) d \Sigma$ la surface mouillée d'unité d'aire de $\sigma$ correspondant aux tubes de filtralion dont les directions interieures $\dot{t} d \Sigma$ sont ainsi sensiblement paralleles à $\bar{n}$. La surface mouillee par ces tubes, dont l'unite d'aire de l'élément de surface $\sigma_{1}$ de normale $\Pi_{1}$ tracéc dans le massif est :

$$
\eta^{\prime}(\pi) \bar{u} \cdot \bar{n}_{1} d \Sigma
$$

Le gradient hydraulique en $M$ suivant la direction $\vec{n}_{1}$ étant $j$, compte tenu du quasi-parallílisme des tubes et par application des résultats anlérieurs $[1.2 .2]$ relatifs à cetle circonstance, le débit filtrant au travers de l'unité d'aire de $\sigma$, le long des tubes de directions intérieures à $d \mathrm{z}$, est :

$$
d q=k \eta^{\prime}(\vec{n}) \bar{n} \cdot \bar{n}_{1} j d \mathrm{~s}
$$

Le débit total, au travers de l'unité d'aire de ", le lons des tubes de toutes directions possibles,

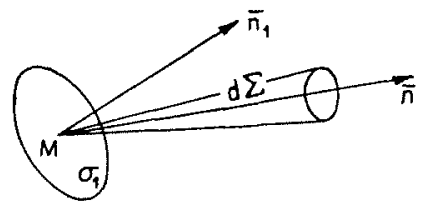

FIt: 3

résulte de l'intégration de di au demi-espace (angle solide $2 \pi$ ) situé par rapport à $\sigma_{1}$ du côté de la normale positive $\bar{n}_{1}$. Soit $(1.1 .18)$ :

$$
\Phi=\frac{p}{\Phi}+y
$$

la charge hydraulique en $\mathrm{M}$, on sait que:

$$
j=-\frac{d \Phi}{d n}=-\left(x \frac{\partial \omega}{\partial x}+\beta \frac{\partial \Phi}{\partial y}+\gamma \frac{\partial \Phi}{\partial z}\right)
$$

Il vient ainsi :

$$
q=-\int_{0}=k \cdot \eta^{\prime} \frac{d \Phi}{d n} \pi \cdot \pi_{1} d \Sigma
$$

Sous le signe d'intégration $k$ et $r_{1}$ sont fonction de l'orientation du tube qu'ils concernent, c'esta-dire de $\pi(\alpha, \beta, \gamma)$, et le résultat de la sommation demeure en définitive fonction de $\pi_{1}\left(\alpha_{1}, \beta_{1}, \gamma_{1}\right)$. Ce qui conduit à poser :

$$
\begin{aligned}
& A=\int_{0}^{2 \pi} k r_{i}^{\prime} x^{2} d \Sigma, \quad B=\int_{0}^{2 \pi} k r_{i}^{\prime} \beta^{2} d L, \\
& \mathrm{C}=\int_{0}^{2 \pi} k \eta^{\prime} \gamma^{2} d \mathrm{~S} \quad \mathrm{D}=\int_{0}^{2 \pi} k \eta^{\prime} \gamma \beta d \mathrm{~s}, \\
& E=\int_{0}^{2 \pi} \operatorname{li} \eta^{\prime} \alpha \eta d \mathrm{v}, \quad \mathrm{F}=\int_{0}^{2 \pi} \ln \eta^{\prime} \beta \alpha d \mathrm{v}
\end{aligned}
$$

et à introduire le tenseur symétrique du second ordre $\mathrm{K}$, de matrice :

$$
=\left|\begin{array}{lll}
A & \text { F } & \text { E } \\
\text { I } & \text { B } & \text { I) } \\
E & \text { J) } & \text { ( }
\end{array}\right|
$$

ou lenseur de perméabilité, par lequel le débil unitaire $q$ s'exprime sous la forme condensée :

$$
q=-\overline{\overline{\mathrm{K}}} \cdot \overline{\operatorname{grad}} \bar{\phi} \cdot \bar{n}_{1}
$$

Notamment, les débits par unité d'aires normales aux axes $\mathrm{O} x, \mathrm{O} y, \mathrm{O} z$, sont respectivement, au point $\mathrm{M}(x, y, z)$.

$$
\left\{\eta_{x}=\mathrm{A} \frac{\partial \Phi}{\partial x}+\mathrm{F} \frac{\partial \Phi}{\partial y}+\mathrm{F} \frac{\partial \omega}{\partial z}\right.
$$

et deux analogues par permutation circulaire.

De (3), par application do la lransformation de GREEs, résulte l'expression du débit sortanl du volume infiniment petil $d ?$ entourant le point M :

$$
d \mathrm{Q}=\cdots \operatorname{div}(\overline{\overline{\mathrm{K}}} \cdot \overline{\text { grad }} \text { 巾) } \mathrm{Q}
$$

L'équation (5) Lraduit la Loi de filtration sous sa forme la plus générale et donne lieu à diverses particularisations.

a) S'agissant d'un massif -.- par exemple conslitué par du sable bien tasse, dont les grains sont lixes el par suite l'indice des vides $m$ constant en chaque point --. siege d'un ceoulement à sur.face libre, elle donne :

$$
\operatorname{div}(\overline{\bar{K}} \cdot \operatorname{grad} \Phi)=0
$$

en tout point de la nappe, et à celte équation 
indéfinie doivent être adjointes deux conditions aux limites exprimant la nullité du débit q normalement au fond imperméable de normale sur lequel repose le sable, et l'égalité de la pression $p$ a la pression atmosphérique $p_{0}$ en tout point de la surface libre (L) de la nappe, surface d'ailleurs inconnue, et variable avec le temps dans toute autre éventualité que celle d'un mouvement stationnaire.

b) Mais sollicité par les charges superficielles et l'action de courant, le massif peut changer de forme - c'est le cas d'une argile qui se consolide - et le module des vides $m$ est fonction du temps. Le volume fluide $m d v$, contenu is l'instant $t$ dans le volume $d$ v gorgé d'eau, augmente de $(\partial \mathrm{m} / \partial t) d t . d v$, pendant la période $d t$ succédant à l'instant $t$, et cette quantité étant précisément égale au débit rentrant $-d \mathrm{Q}$ dans $d v$ pendant ce temps, il vient par (5) l'équation indéfinie de l'écoulement :

$$
\frac{\partial m}{\partial t}-\operatorname{div}(\overline{\bar{K}} \cdot \overline{\text { grad } \Phi)}=0
$$

c) Plus généralement, que les pores de $d ?$ soient ou non gorgés d'cau, désignant par Ji d ? lo volume de celle-ei it l'instant $t$, on al :

$$
\begin{array}{ll}
\partial \mathfrak{i} & \cdots \operatorname{div}(\overline{\mathrm{K}} \cdot \operatorname{grad} \overline{\mathrm{p}})=0 \\
\partial t & \cdots
\end{array}
$$

d) Les moubements permanents de filtration pour lesquels vitesses et pressions demeurent indépendantes du temps méritent enfin une attention spéciale. En tout point $\mathrm{M}(x, y, z)$, la charge w est fonction, à l'exclusion du temps $t$ des seules coordonnées $x, y, z$. La vitesse brute de filtration est toujours $V=-\overline{\mathrm{K}}$. grad $\Phi$ et l'équation de continuite traduisant l'invariance du flux sortant de l'ilement de volume s'écrit:

$$
\operatorname{div}(\overline{\mathrm{K}} \cdot \overline{\text { grad } \Phi})=0 \text {. }
$$

\section{HoMOgéNÉITÉ ET ISOTROPIE.}

Les coordonnées du tenseur de perméabilité el ses directions principales dépendent en toute généralité des coordonnées $x, y, z$, du point $M$ où est considéré l'écoulement. Une circonstance remarquable est celle d'un milieu homogène pour lequel ces grandeurs sont en tout point les mèmes, ce qui entraine d'évidentes simplifications tenant a l'existence d'axes principaux de directions fixes, par rapport auxquels $\overline{\mathrm{K}}$, constant dans tout le massif, prend la forme réduite diagonale :

$$
\ddot{\bar{K}}=\mid \begin{array}{lll}
A & 0 & 0 \\
0 & B & 0 \\
0 & 0 & 0
\end{array} \|
$$

Il vient alors :

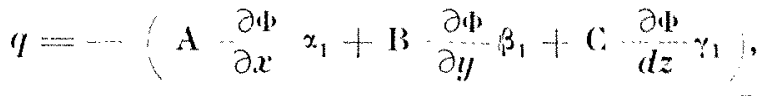

$$
\begin{aligned}
& d Q=\cdots, A \frac{\partial^{2} w}{\partial x^{2}}+B \frac{\partial^{2} w}{d y^{2}}+C \frac{\partial z^{2}}{\partial z^{2}} ; d v .
\end{aligned}
$$

Un autre cas particulier important concerne les milieux isotropes, tels que K et $r_{\text {y }}$ soient, en un point $M$ donné, les mêmes pour toutes directions, c'est-id-dire independants de $\mu(\alpha, \beta, \gamma)$. Le tenseur $\overline{\overline{\mathrm{K}}}$ prend alors une forme simple, puisque ses coordonnces :

$$
A=k+r_{1} \int_{0}^{-2 \pi} x=d=, \quad \text { B. } \quad \text { ： }
$$

sont égales, tandis que :

$$
\mathrm{D}=\mathrm{kin} \int_{1+\pi}^{\cdots+\pi} \gamma \mathrm{a}, \quad \mathrm{E}, \quad \mathrm{F}
$$

sont nulles, en applieation de deux proprietes connues of quasi évidentes des fonctions orthogonales. Le calcul de la valeur commune des trois premieres seffectue dans le systime de coordonnées sphériquess $(r=1,0,0)$ de pòle $M$, el cette quantité ou coefficient de permerabilits du milien isotrope a pour valeur :

$K=k \cdot r^{\prime} \int_{11}^{2 \pi} d ? \int_{1}^{\pi / 2} \cos ^{2} \theta \sin \theta d \theta-\frac{2}{3} \pi k r_{1}^{\prime}$.

mais sa délermination a partir de la formule cidessus présente les mêmes difficultés que celles de $k$ et $r_{i}^{\prime}$ (ef. [ $\left.\begin{array}{lll}1 & 2 & 2\end{array}\right]$ et est finalement demandie a l'expérience.

Les débits définis au précedent article ont ainsi, dans le cas de l'isotropie du milicu, respectivement pour expressions :

$$
\begin{gathered}
q=: \mathrm{K} \overline{\operatorname{grad} \Phi \cdot \mu_{1}} \\
d \mathrm{Q}=-\operatorname{div}(\mathrm{K} \operatorname{grad} \Phi) d v
\end{gathered}
$$

Enfin, la double circonstance d'une isotropie homogene, si fríquemment évoqué par les inginieurs comme approximation suffisante pour leurs besoins, implique la constance du coefficient de perméabilité scalaire $K$ dans tout le mas- 
sif, d'on résulte l'expression du débit sortant du volume $d \mathfrak{v}$ :

$$
l Q=-K \frac{\partial^{2} \Phi}{\partial x^{2}}+\frac{\partial^{2} \Phi}{\partial y^{2}}+\frac{\partial^{2} \Phi}{\partial z^{2}} d w
$$

ef la condition sur le contour imperméable (S) de normale $\bar{n}(\alpha, \beta, \gamma)$ :

$$
\approx \frac{\partial \Phi}{\partial x}+\beta \frac{\partial \Phi}{\partial y}+\because \frac{\partial \Phi}{\partial z}=0
$$

\section{ACTION DF COURANT.}

Laction exercée par le courant sur les grains du massif perméable entre lesquels il filtre se détermine comme en $[1.2 .3]$ où sont mis en évidence comme sollicitant le volume élémentaire $d v$

- laction archimédienne:

$$
\text { का }(1-m) \overline{\text { gata } !} \text { d ? }
$$

-.. l'action de courant:

$$
\text { - } \overline{\text { grad }} d v
$$

soit pour l'action totale :

$$
\overline{d A}=[-\bar{\omega} \overline{\operatorname{grad} \Phi}+\bar{\omega}(1-m) \operatorname{grad} y] d \vartheta
$$

indépendante du tenseur de perméabilité.

\section{RÉsumé ET CONCLUSIONS.}

Les développements ci-dessus supposent ainsi les massifs naturels formés de grains, réductibles, en ce qui concerne l'écoulement de filtration, au schéma suivant. Disposant d'un très petit volume matériel continu $d \vartheta$ et d'un très grand nombre de fines aiguilles, à l'aide de chacune de celles-ci on transperce le promier d'une manière quelconque un très grand nombre de fois. Le résultat de l'opération est une des particules macroscopiques dont la juxtaposition constitue le volume filtrant $\mathfrak{v}$ soumis au calcul. Ainsi à la structure naturelle granulaire, a été substitué, pour les besoins de l'analyse, un continu matériel percé de tubes. Cet artifice n'est pas sans soulever quelques objections théoriques, mais l'expérience en justifie dans une certaine mesure la légitimité. Ceci étant, l'établissement des équations générales régissant le champ des vitesses et celui des actions hydrodynamiques internes d'un tel massif a nécessite l'introduction successive :

a) D'un coefficient de filtration $k(\vec{n})$ et de l'airc movillée unitaire $r^{\prime}(\bar{i})$ relatifs en tout point $M$ in chaque direction de l'espace:

b) Du tenseur $\overline{\bar{k}}$, dont les coordonnées, par suite d'un changement de notations (qui s'explique de lui-mème, sont contenues dans l'expresession :

$$
K_{i j}=\frac{1}{2} \int_{0}^{*} k \eta^{\prime} \alpha_{i} x_{j} d_{\sigma}
$$

l'intégrale étant étendue à la sphère de centre M et de rayon unité.

Cette grandeur fondamentale, que nous arons proposé autrefois d'appeler tenseur de perméabilité, donne licu à interprétation intéressante. Elle n'est autre en effet que le tenseur d'inertie relatil à son centre d'une simple couche sphérique de rayon unité et de densité $(1 / 2) k \cdot \eta^{\prime}$. L'ellipsoüde d'inertie de cette distribution est indépendant du point considéré du massif si ce dernier est homogène, c'est une sphère s'il est isotrope. Dans la plupart des cas usuels d'une constitution ayanl mis en ouvre la seule gravité, il est de révolution autour de son axe vertical. $\Phi$ désignant en chaque point le potentiel de l'écoulement ou charge au sens de Bernoular, les propriétés du champ des ritesses et du champ des actions hydrodynamiques sont résumées formellement par la valeur du débit filtrant au travers de l'unité de volume, soit :

$$
-\operatorname{div}(\overline{\overline{\mathbf{K}}} \cdot \overline{\operatorname{grad}} \Phi),
$$

et par celle de l'action exercce par le courant sur la uème portion du massif, soit :

$$
-\overline{\operatorname{grad}} \Phi .
$$

indépendante de la constitution de celui-ci et de la viscosité du liquide en mouvement.

Il convient, en outre, de noter que tout ceci suppose essentiellement :

a) Le régime de filtration, lent, uniforme ou quasi uniforme, ce qui revient à admettre une limile superieure du gradient hydraulique et une limite supérieure de ses variations temporelles, au-delà desquelles cette double circonstance n'est pas réalisée;

b) Que la proportion de l'eau morte, entourant les grains, à l'eau circulante, qui semble bien devoir dépendre du gradient hydraulique, demeure faible $[1.2 .3]$, ce qui limiterait inférieurement cette dernière quantité.

Sous ces réserves, c'est-à-dire dans la plupart des iventualités usuelles, les résultats établis sont propres à représenter convenablement le phénomène en cause. 


\section{IV. - APPLICATIONS}

La détermination de l'écoulement de l'ean dans le sol a donne lieu à dimporlants travax dont les résultats sont exposés dans de nombreux ouvages, notamment dans ceux cités en référence auxquels le lecteur se reportera éventuellement. Il nous a néanmoins semble utile de classer et de décrire ici les méthodes qui y sont couramment mises en ouvre et par lesquelles à une analyse souvent inacessible sont substitués de fructueux procédés dapproximation. A cette fin, nous envisagerons trés généralement, et sauf exception spécifiée, des mouvements plans, en distinguant les ecoulements non permanents des écoulements slationnaires, les écoulements en charge des écoulements à surface libre.

\section{a) Mouvements permanents}

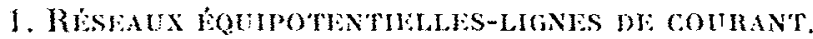

Sous l'hypothese de la permanence de l'écoulement, vitesse et pression sont indépendantes du lemps, et la charge hydraulique i en tout point est fonction des seules coordonnées de celui-ci.

Dans l'hypothise la plus générale de lanisoIropie, la vitesse brute de filtration (1.2.3) est :

$$
\overrightarrow{\vec{V}}=-\overline{\overline{\mathrm{K}}} \cdot \overline{\text { grad } \Phi}
$$

ef l'épuation de continuité lraduisant linvarianec temporelle du flux sortant de l'elément de polume s'écrit $(1.2 .10)$ :

$$
\operatorname{div}(\overline{\bar{K}} \cdot \overline{\operatorname{arad}} \mathbf{\mathrm { I }})=0
$$

L'alude des monrements en cause sera limilée au cas dun phénomène plan rapporté aux axes O.x.Oy (Oy, verticale ascendante) et d'un massif homogene de directions principales parallèles aux axes de cooldonnées. Cette circonstance, d'un réel inléret pratique, concerne en particulice les infiltrations dans le corps des digues en terre, les inliastruclures de barrage, autour des parafonilles de ces ourrages, ef généralement a lravers lout massif, indéfini le long d'une direction horizonlale $\mathrm{Oz}$, dont le mode de constitution par sédimentation (massifs naturels) ou compactage (massifs artificiels) a mis en jeu la seule gravifi.

Soient $u$ et $v$ les coordonnes de $Y$, A et B celles du tenseur de perméabilité $K$, mis sous forme diagonale, qui ne sont autres que les coefficients de perméabilité horizontal el vertical du massif. Le mouvement de filtration est régi par les equations :

$$
\begin{array}{r}
\|=\ldots \frac{\partial \omega}{\partial x}, \quad n=\ldots \mathrm{B} \frac{\partial \Phi}{\partial y} \\
A \frac{\partial^{2} \Phi}{\partial x^{2}}+B \frac{\partial * \omega}{\partial y^{2}}=0
\end{array}
$$

Les lignes :

$$
\text { W }(x, y)=h(h \text { paramile } \Phi=(p / \omega)+l)
$$

sont les equipotentielles du champ de lillation. Les lignes de courent $\Psi(x, y) \cdots k$ ( $k$ parametre) sonl definies par :

$$
n \frac{\partial \Psi}{\partial x}+n \cdot \frac{\partial y}{\partial y}=u
$$

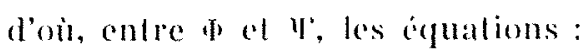

$$
\frac{\partial \Psi}{\partial x}=13 \frac{\partial \psi}{\partial y}, \frac{\partial \Psi}{\partial y}=A \cdots \frac{\partial \psi}{\partial x}
$$

compatibles avec léquation de continuile (2). Equipotenticlles el lignes de courant forment un rescau dont vaici les principales proprieles. Soil l'une des malles de celuimei, constilue par l'infersection des dewx equipolentielles $(h, h \ldots$ is $h)$

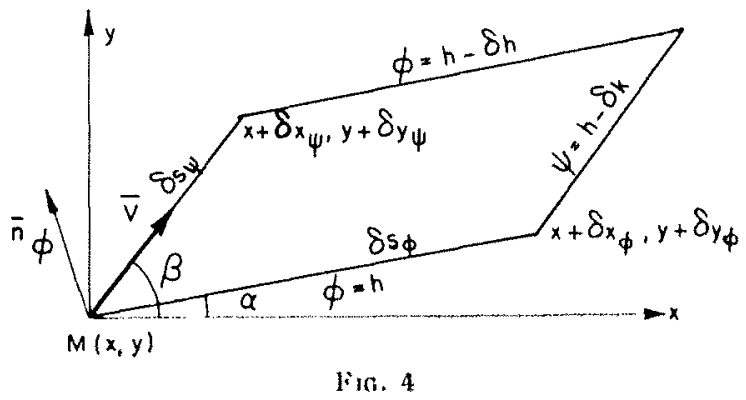

al des deux lignes de courant (k, $k-z$ - $k$ ) (lig. 4).

le flux ou debil circulant entre les deux lignes

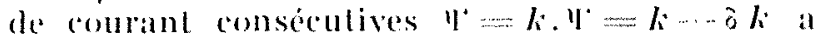
pour valeur :

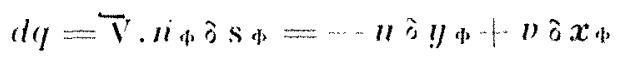

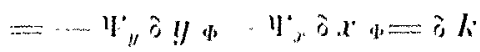

$1 /$ est eqal a la difference de cole de ces lignes.

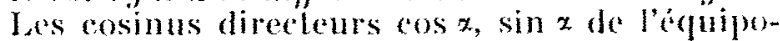
lentielle $w=h$, en $M$, coss et sing de la ligne de courant $\Psi^{\circ}=k$ au mème point, proporlionnelles respectivement a -... $\Phi_{x}$ of $\Phi_{\|}$pour les premices, à 11 et $v$ pour les sceonds, satisfont à :

$$
\frac{\cos \alpha \cos \beta}{A}+\frac{\sin \alpha \sin \theta}{B}=0
$$

Ainsi, équipotentielle el ligne de comrant du point M sont conjugués relativement a lellipse de centre $\mathrm{M}$ et déquation : 


$$
\frac{x^{2}}{\mathrm{~A}}+\frac{y^{2}}{\mathrm{~B}}-1
$$

Ia definition du réseau s'achève par colle de la longueur d'arc d'équipotentielle os $s_{\text {q }}$ comprise entre les lignes de courant cotées $k$ et $k \cdots-j k$.

On a :

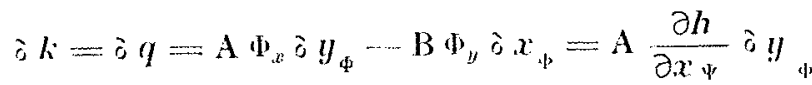

$$
\begin{aligned}
& \text { B } \frac{\partial h}{\partial y_{w}} \delta x_{w}
\end{aligned}
$$

soil :

$$
\partial k=-\partial h \frac{\partial s_{\Phi}}{\partial s_{\Psi}}\left(A \frac{\sin \alpha}{\cos \beta}-B \frac{\cos \alpha}{\sin \beta}\right),
$$

d'où, par (4):

$$
\delta s_{\Downarrow}=\frac{1}{\sqrt{A B}} \frac{\partial k}{\partial h} \partial s_{\psi}
$$

Le cas de l'isotropie mérite une attention parliculiere. Dans cette éventualite, $A=B=K$, les equations (1) et (2) deviennent:

$$
\begin{gathered}
u=-\mathrm{K} \frac{\partial \Phi}{\partial x}, \quad v=-\mathrm{K} \frac{\partial \Phi}{\partial y} \\
\frac{\partial^{2} \Phi}{\partial x^{2}}+\frac{\partial \cdot \Phi}{\partial y^{2}}=0
\end{gathered}
$$

Le problème se réduit à la recherche d'une fonction hamonique is satisfaisant aux conditions aux limites imposées sur lesquelles nous reviendrons. La solution est indépendante du coefficient de perméabilité $K$ et il en est de même du réseau équipotentielles-lignes de courant qui devient orthogonal et dont les arcs satisfont à :

$$
\partial s_{w}=\frac{1}{\mathrm{~K}} \frac{\partial k}{\partial h} \delta s_{\Psi}
$$

Il est d'ailleurs loisible de réduire au cas isotrope celui de l'anisotropie, puisque l'affiniti :

$$
\mathrm{X}=\frac{x}{\sqrt{\mathrm{A}}}, \quad \mathrm{Y}=\frac{y}{\sqrt{\mathrm{B}}},
$$

transforme les équations (1) et (2) en :

$$
\begin{gathered}
u=-\sqrt{\mathrm{A}} \frac{\partial \Phi}{\partial \mathrm{X}}, \quad v=\cdots-\sqrt{ } \mathrm{B} \frac{\partial \Phi}{\partial \mathrm{Y}} \\
\frac{\partial^{2} \Phi}{\partial \mathrm{X}^{2}}+\frac{\partial^{2} \Phi}{\partial Y^{2}}=0 .
\end{gathered}
$$

Celle remarque permet de limiler les dévelop- pements qui vont suivre à la cireonstance typique de la filtration isotrope.

\section{ECOVLEMENTS EN CHARGE.}

Nous désignons ainsi ceux qui intéressent l'étendue d'un massif perméable limité par des régions imperméables cojacentes au premier. Les ares de frontière sont lignes de courant. C'est le cas d'une nappe captive lelle celle représentéc ci-dessous (fig. 5).

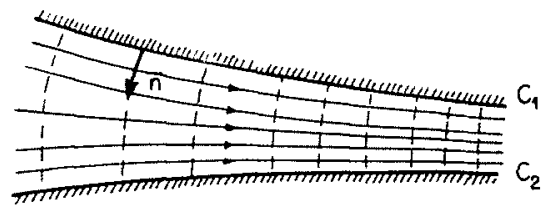

Fig, 5

Le probleme consiste à déterminer la fonction harmonique $\Phi(x, y)$ satisfaisant au contour C (lignes $C_{1}$ et $\left.C_{2}\right)$ à la condition $(d \Phi / d n)=0(\pi$, normale au contour). C'est là un probleme de Drnichret. Celui-ci étant résolu, la vilesse est connue en tout point comme $K$ grad $\Phi$, et la pression résulte de la définition de la charge $\Phi=(p / \omega)+y$, qui remplace ici l'équation de LAGRANGe de l'hydrodynamique des courants plans irrotationnels. Les procédés théoriques (potentiel complexe) et expérimentaux (cuve rhcoélectrique) employés pour le résoudre sont connus et nous n'y insisterons pas en nous bornant a présenter deux exemples simples et particulièrement caractéristiques.

\section{a) Drainage d'une nappe captive.}

Soit une nappe indéfinie vers le bas, limilée vers le haut par un plan horizontal $x \circ z$ au-dessus duquel règne un massif imperméable. Celle nappe est animée à l'infini d'une vitesse uni-

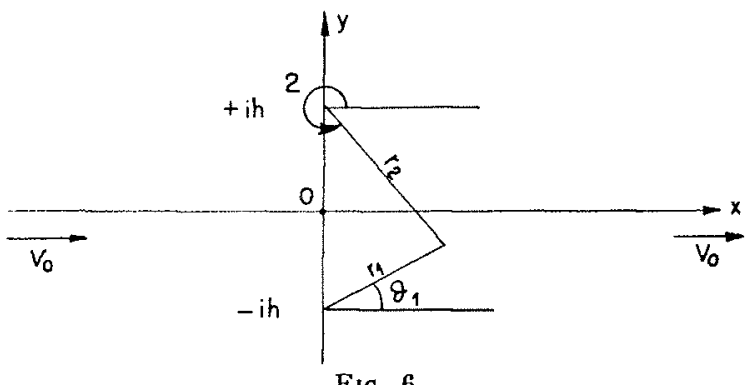

FIG. 6

forme horizontale parallele a $o x$, de grandeur $V_{0}$ et comporte un drain rectiligne indéfini horizontal parallèle à $o z$, calé à la cote - $-h$, de débit $Q$ (fig. 6). Il s'agit de déterminer les caractéristiques de l'ícoulement dans le massif perméable. 
Le drain de fabble rayon est assinilable it un puits hydrodynamique d'affixe - ih, de débit $Q$, et la méthode des images conduit à assigner atu potentiel complexe l'expression $f(z)$ de la variatble $z=x+i y$ :

$$
f(z)=-\frac{V_{0}}{\mathrm{~K}} z-\frac{\mathrm{Q}}{2 \pi} \log (z-+-i h)(z-i-i h)
$$

S'en déduisent les équipotentielles el les lig̣nes de courant d'équations respectives :

$$
\begin{aligned}
\Phi=\frac{V_{0}}{\mathrm{~K}} x-\frac{\mathrm{Q}}{2 \pi} & \log r_{1}, r_{2}, \\
\Psi & =\frac{V_{0}}{\mathrm{~K}} y-\frac{\mathrm{Q}}{2 \pi}\left(0_{1}+0_{2}\right) .
\end{aligned}
$$

ainsi que l'expression de la vitesse complexe $w$ :

$$
w=u-i v=V_{0}-\frac{\mathrm{Q}}{2 \pi}\left(\frac{1}{z+i h}+\frac{1}{z-i h}\right)
$$

Dans le cas particulier d'une nappe au repos à l'infini $\left(V_{0}=0\right)$, le réseau équipotentielles-lignes de courant comporte une famille d'ovales de Cissinf évanescente en - ih, et unc lamille d'hyperboles d'asymptote ox (fig. 7).

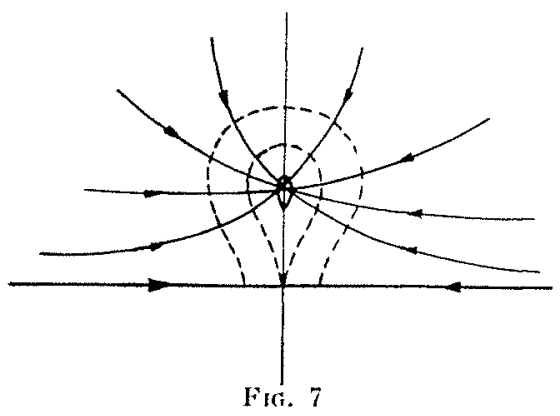

\section{b) Filtration sous un ridean de palplanches.}

Le rideau vertical $A B$ est foncé jusqu'à la cote -- $h$ dans un sol perméable indéfini de perméabilité K. De part et d'autre, l'eau en équilibre présente une dénivelée $H$ (fig. 8).

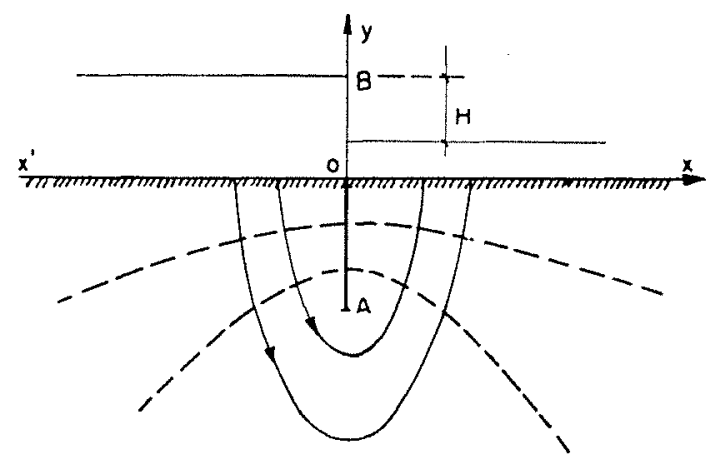

Fifi. 8
Lécoulement de filtration qui sélablit dams le massif admet comme ligne de courant imposie le segment od (à l'amonl), AO (à l'aval) el comme equipolentielles les demi-droiles ox el ox entre lesquelles la difference de potentiel est is $\mathrm{H}$. On salisfail a ces conditions par le polentiel complexe:

$$
f(z)=\frac{\pi H}{\pi} \arccos \frac{z}{i h}
$$

par lequel :

$$
\begin{aligned}
& x=h \sin \frac{\pi \mathrm{H}}{\pi \mathrm{H}} \operatorname{sh} \begin{array}{c}
\pi \mathrm{y} \\
\pi \mathrm{H}
\end{array} \\
& y=h \cos \frac{\pi \mathrm{\omega}}{\pi \mathrm{H}} \text { ch } \frac{\pi \mathrm{H}}{\pi \mathrm{H}}
\end{aligned}
$$

Les lignes de courant sont les demi-cllipses d'équation :

$$
\operatorname{sh} 2 \frac{x^{2}}{\pi \mathrm{H}}+\frac{y^{2}}{\operatorname{ch}: \frac{\pi \Psi^{2}}{\pi \mathrm{H}}}=h
$$

Les equipotentielles sont les quarts dhyperboles homolocales des précédentes (foyer $A$ ) d'equation :

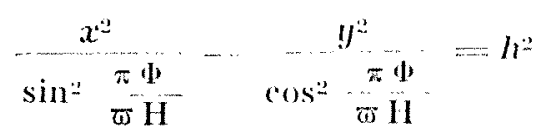

\section{Ecollements a surpace Labi:.}

C'est le cas des nappes phréatiques. Tres genéralement la fillration seffeclue au-dessus du toil (C) d'un massif impermeable, lout en etant limilée vers le haul par une lighe d'eau (S), fixe en regime slationnatre, mais a priori inconnue (fig. 9). Il s'agil encore de délerminer le pulen-

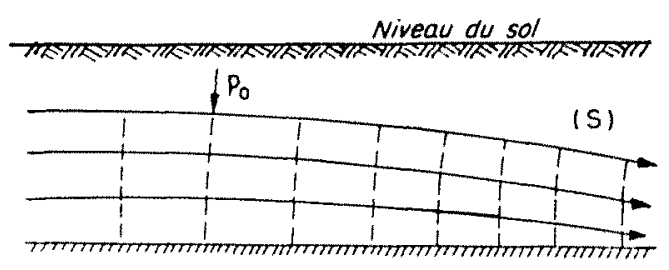

Fig. 9

tiel harmonique of $(x, y)$. Mais l'existence de la ligne deau. ligne de comint le longs de laquelle la pression egale la pression almosphérique $p_{0}$, introduit les nouvelles conditions atux limites:

$$
\frac{d \Phi}{d n}=0 \quad, \quad \omega=\frac{P_{3}}{\mathbb{\omega}}+\eta /
$$


le longs de cette courbe (S), dont l'ordonnée $y(x)$ est une inconnue du problème. L'analyse s'avère impuissante pour aborder celui-ci et il faut se résoudre à l'emploi de méthodes approchées qui semblent proceder de trois circonstances distinctes.

1" L’écoulement résulte de l'introduction, dans la nappe au repos ou de mourement connu, de quelques singularités aflectant suffisamment peu la ligne d'ean primitive pour que celle-ci puisse être conservée. La méthode du potentiel complexe peut encore être appliquée;

2" L'intuition ou quelques tâtonnements dirigés par celle-ci permet le tracé de la ligne d'eau qui se trouve graduce en charge et sert de base de départ pour le tracé du réseau quadratique dont les équipotentielles doivent aboutir normalement aux lignes de courant imposies. C'est l'extension aux phénomènes de filtration de la méthode de Prasir familiere aux hydrauliciens;

3" I,es niveaux d'alimentation ef de restitulion chant peu différents, la lighe d'ean $y=y(x)$ présente de faibles pentes, et les iquipolentielles qui la quittent normalement se confondent avec des segments rectilignes verlicaux. Lat conservation du débil filtrant a travers ces derniers fixe l'équation diffirenticlle de la ligne (S).

\section{a) Determinalion in silu de la perméabilité d'un sol}

Dans le sol perméable de profondeur indéfinie, la nappe d'eau de surface libre xoz lorizontale est au repos. Une crépine sphérique de rayon : est foncé dans celle-ci à la profondeur $h$, relativement grande par rapport à o (fig. 10) et per- met d'injecter dans le terrain un débit d'eau dont est mesurce la valeur $Q$ en régime permanent. Si la surtace libre $x o y$ n'est pas sensiblement modifice par l'écoulement ainsi déterminé, le potentiel dont dérive la vitesse de ce dernier

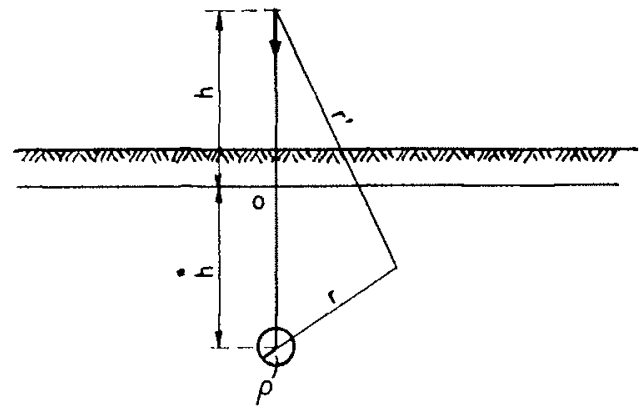

Fig. 10

est (methode des images, fonction harmoniquelype de lespace) :

$$
\mathrm{W}=\frac{\mathrm{Q}}{4 \pi \mathrm{K}}\left(\frac{1}{r}+\frac{1}{r^{\prime}}\right)
$$

Il s'annule à l’intini et est équiralent à $\mathrm{Q} /(4 \pi \mathrm{K}$ ) al voisinage de la crepine. La dillerence des lectures faites sur deux tubes piézométriques établis l'un au voisinage de la crépine, l'autre tres loin de celle-ci, foumira cette hauteur d'où résulte la valeur du coefficient de perméabilité $K$. La méthode décrite implique unc evidente contradiction, puisque le plan xoy y est simultanément considéré comme une équipotentielle (charge constante) el comme une surface de courant (méthode des imases). Ceci n'a pas lieu de nous surprendre : en fait, la surface

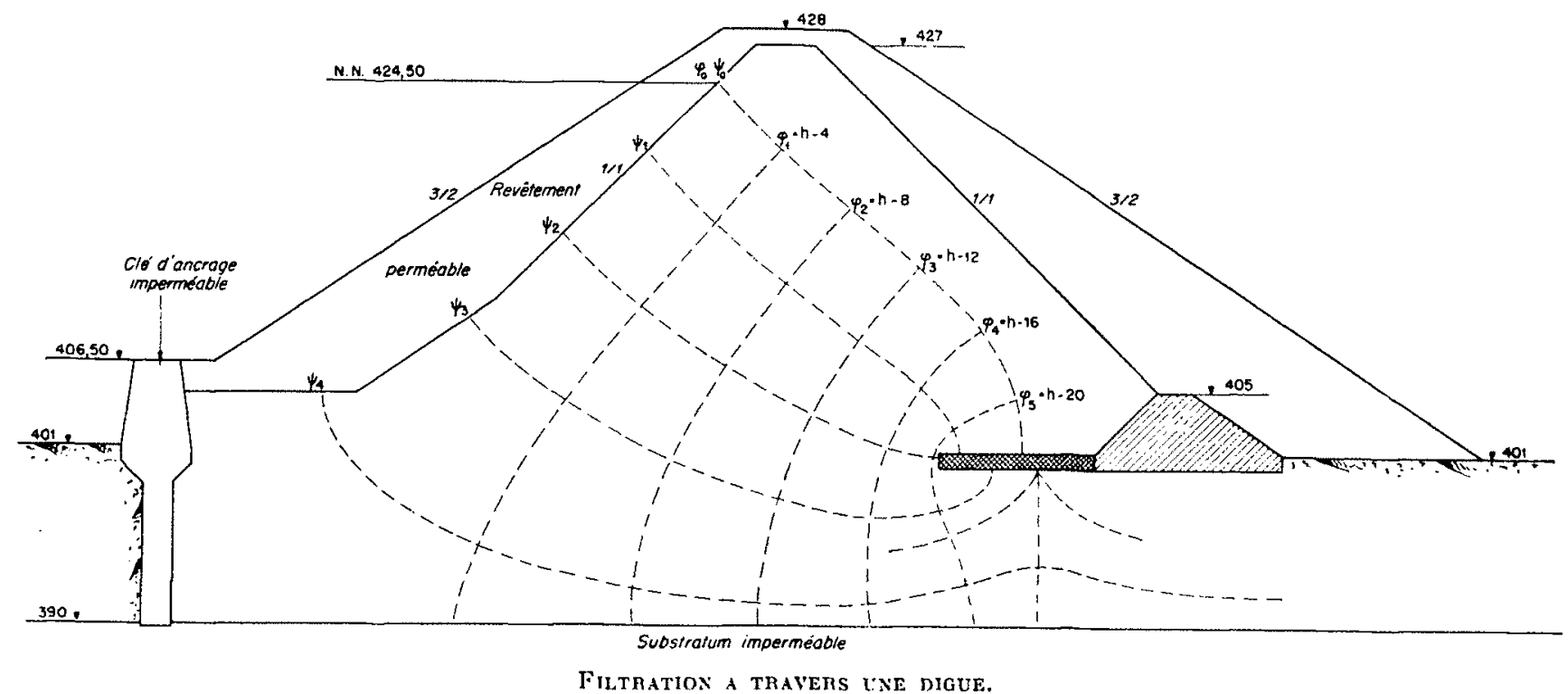


libre se gonfle manifestement à la verticale de la source pour se raceorder asymptotiquement avec le plan horizontal xoy arec lequel nous l'avons délibiriment confondue.

\section{b) Fillralion it travers une dique.}

La planche ci-contre figure la coupe en travers d'un barrage de retenue établi sur terrain imperméable $(+390)$. La filtration $y$ intervient entre le niveau amont $(+424,50)$ el le filtre aval (401). La ligne d'eau quittant normalement le talus amont en A pour aboutir en $B$ normalement au filtre a été tracée arbitrairement et graduée en charge. Les condilions aux limiles auxquelles il a fallu satisfaire sont évidenles : le parement amont OA et le contour du filtre sont deux équipotentielles, l'horizonlate (390) est ligne de cottrant. On observerat la réflexion des lignes do courant sur la face inféricure du filtre. Le coefficient de perméabilité de la digue étant $K=6.10^{-i} \mathrm{~m} / \mathrm{h}$, le débit recueilli par le drain ressort ì $Q=10^{-5} \mathrm{~m} \% / \mathrm{h}$.

\section{c) Mouvement laminaire plan des eam sonteraines.}

La fillation s'efreclue dans le plan vertical XOY (OY, verticale descendante) au-dessus d'un massif impermcable dont le toit rectiligne fait l'angle $i$ aver l'horizontale (fig. 11). Lapproxima-

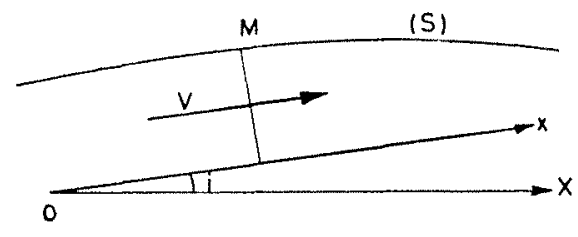

Fisi, 11

lion, justifiée par la fable inclimaison de la ligne d'eau (S), consiste à supposer toutes les lignes de courant parallèles à cet axe et à identifier, par conséquent, les equipolentielles à des segments rectilignes de direction oy.

Soit $M(x y)$ un point de la ligne d'eau, $m$ sa projection sur ox. En M regne la pression atmosphérique $p_{0}$ et la charge est :

$$
\Phi=\frac{p_{0}}{\omega}+\mathrm{Y}
$$

avec :

$$
\mathrm{Y}=x \sin i+y \cos i
$$

elle conserve celte valeur le long de la droite m M, c'est-à-dire pour $x$ donné. On en déduit la vitesse moyenne brute de filtration :

$$
V=K\left(\sin i+\cos i \frac{d y}{d x}\right) \text {, }
$$

et le débil mnitaire suivanl la direction de laxe () $z$.

$$
Q=-K y\left(\sin i+\cos i \frac{d y}{d x}\right)
$$

La conservation de celui-ci entraine lépualion différentielle de la ligne d'ean:

$$
\cos i \frac{d y}{d x}+\frac{\mathrm{Q}}{\mathrm{k} y}+\sin i \cdots 01
$$

dont linlegration est immediale.

Soil lexemple deune fillration permantenle andessus d'un massif imperméahle horizomlal entre deux bassins a niveau comslants $H$ ol $h$, dislants de L. (fig. 12).

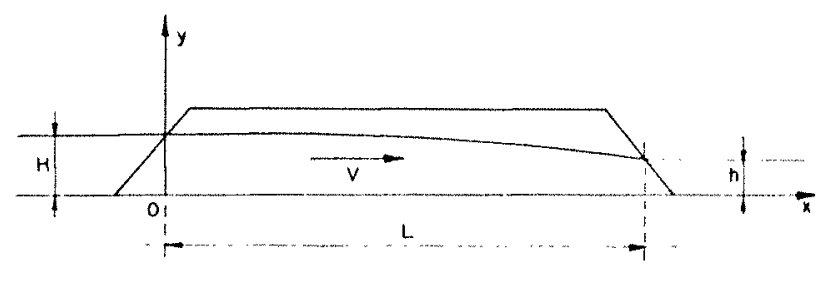

Fili. 12

Le dóbil fillrant est:

$$
\text { Q }=-\frac{\mathrm{K}}{2 \mathrm{I}}\left(\mathrm{H}^{2} \ldots h^{2}\right) \text {, }
$$

el la ligne d'eau a pour équation:

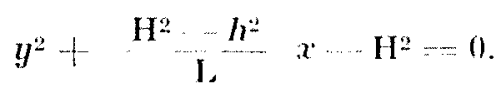

Ces résultals sont liciles dams la mesure où L/H est grand. L'equation de (S) ast de loule manière dépourve de sens au voisinago des seclions d'extrémités $x=0, x=\mathrm{I}$.

\section{b) Mouvements non permanents}

Il s'agit d'étudier la filtration déterminé par des conditions dalimentation ot de restilution variables dans un massif perméable a struclure invariable. Un exemple mettra en lamiere la complexité des problèmes de l'espèce. 


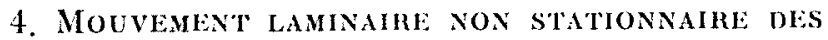
FAUX SOUTERRAINES.

Reprenons le cas traité ci-dessus [3.c] en ne supposant plus la permanence du mouvement. Le débit, unitaire suivant $o z$, filtrant parallelement à ox entre cette ligne de courant et la ligne d'eau variable $y=y(x, t)$ est à l'instant $t$ :

$$
\mathrm{Q}=-\mathrm{K} y\left(\sin i+\cos i \frac{\partial y}{\partial x}\right)
$$

D'autre part, désignant comme ci-dessus le module des vides du massif par $m$, il vient pour l'équation de continuité :

$$
m \frac{\partial y}{\partial t}+\frac{\partial Q}{\partial x}=0
$$

L'élimination de $Q$ entre les deux précédentes donne l'équation aux dérivées partielles du second ordre :

$$
\frac{\partial y}{\partial t}=\frac{K}{m}\left[\sin i \frac{\partial y}{\partial x}+\cos i\left(\frac{\partial y}{\partial x}\right)^{2}+\cos i \cdot y \frac{\partial^{2} y}{\partial x^{2}}\right]
$$

dont l'intégrale n'est connue que pour quelques cas particuliers qui vont ètre rapidement évoqués:

a) Si la pente de la ligne d'eau $\partial y / \partial x$ demeure faible devant $\sin i$, l'équation en question se riduit à :

$$
\frac{\partial y}{\partial t}=\frac{K}{m} \sin i-\frac{\partial y}{\partial x}
$$

et admet l'intégrale générale:

$$
y=f\left(x+\frac{K t}{m} \sin i\right)
$$

exprimée à l'aide de la fonction $f$ satisfaisant aux conditions d'alimentation, c'est-à-dire pre- nant pour $\boldsymbol{x}=\mathbf{0}$ la forme donnée $\mathrm{F}(\boldsymbol{l})$. Elle fait apparaître une célérité $[(\mathrm{K} / \mathrm{m}) \sin i]$ pour la propagation de la perturbation qui s'effectue par ondes planes.

b) Généralement, la pente du fond étant faible ( $\sin i \# i ; \cos i \# 1)$, cette approximation n'a plus de sens, $y$ demeure voisin du niveau amont initial $H$, et la pente de la ligne d'eau demeurant petite, $(\partial y / \partial x)^{2}$ est négligeable dans l'équation aux dérivées partielles qui s'écrit :

$$
\frac{\partial y}{\partial t}=\frac{\mathrm{K}}{m}\left(i \frac{\partial y}{\partial x}+\mathrm{H} \frac{\partial^{2} y}{\partial x^{2}}\right)
$$

Elle admet la solution particulièe de BocssiNESQ :

$$
y=-\mathrm{M} e^{-(k / m)\left(4 \pi / \mathrm{S}^{2}\right) \mathrm{H} t} \cos \frac{2 \pi}{\mathrm{S}}\left(\frac{\mathrm{K} i t}{m}+\mathrm{N}-x\right)
$$

qui correspond à la propagation d'un mouvement sinusoïdal amorti avec la célérité $\mathrm{K} i / \mathrm{m}$.

c) Enfin, l'écoulement sur fond horizonlal $(i=0)$ est régi par l'équation :

$$
\frac{\partial y}{\partial t}=\frac{K H}{m} \frac{\partial^{2} y}{\partial x^{2}}
$$

dont une solution particulière est:

$y=M \cos \left(-\frac{2 \pi t}{\mathrm{~T}}+\sqrt{\frac{\pi m}{2 \mathrm{KH} T}} x\right) e-\sqrt{[(\pi m) / 2 \mathrm{KH} T)} x$

Cette dernière montre qu'une oscillation sinusoïlale de période $\mathrm{T}$ intervenant dans le bassin amont $(x=0)$ se propage sous forme d'oscillation amortie avec la célérité $\sqrt{ } 2 \pi \mathrm{KH} /\left(\mathrm{m}^{r} \mathrm{~T}\right)$. Le theorème de Fourien étend ce résultat à toute perturbation périodique. Bien entendu, les réserves formulées au sujet du mouvement permanent sont valables pour la circonstance qui vient d'être envisagée.

(A suivre.) 